\begin{tabular}{|c|l|}
\hline Title & Locally and Globally Coupled Oscillators in Muscle \\
\hline Author(s) & Sato, Katsuhiko; Kuramoto, Yoshiki; Ohtaki, Masako; Shimamoto, Y uta; Ishiwata, Shin'ichi \\
\hline Citation & $\begin{array}{l}\text { Physical Review Letters, 1111(10), 108104 } \\
\text { https://doi.org/10.1103/PhysRevLett.111.108104 }\end{array}$ \\
\hline Issue Date & 2013-09-06 \\
\hline Doc URL & http://hdl.handle.net/2115/64299 \\
\hline Rights & ○ 2013 The American Physical Society \\
\hline Type & article \\
\hline File Information & matj79_PhysRevLett.111.108104.pdf \\
\hline
\end{tabular}

Instructions for use 


\title{
Locally and Globally Coupled Oscillators in Muscle
}

\author{
Katsuhiko Sato, ${ }^{1, *}$ Yoshiki Kuramoto, ${ }^{2}$ Masako Ohtaki, ${ }^{4}$ Yuta Shimamoto,${ }^{3}$ and Shin'ichi Ishiwata ${ }^{4,5, \dagger}$ \\ ${ }^{1}$ RIKEN Center for Developmental Biology, 2-2-3 Minatojima-minamimachi, Chuo-ku, Kobe 650-0047, Japan \\ ${ }^{2}$ International Institute for Advanced Studies, Kyoto 619-0225, Japan \\ ${ }^{3}$ Laboratory of Chemistry and Cell Biology, Rockefeller University, 1230 York Avenue, New York, New York 10065, USA \\ ${ }^{4}$ Department of Physics, Faculty of Science and Engineering, Waseda University, 3-4-1 Okubo, Shinjuku-ku, Tokyo 169-8555, Japan \\ ${ }^{5}$ Waseda Bioscience Research Institute in Singapore (WABIOS), Singapore 138667, Singapore
}

(Received 12 January 2013; published 5 September 2013)

\begin{abstract}
At an intermediate activation level, striated muscle exhibits autonomous oscillations called SPOC, in which the basic contractile units, sarcomeres, oscillate in length, and various oscillatory patterns such as traveling waves and their disrupted forms appear in a myofibril. Here we show that these patterns are reproduced by mechanically connecting in series the unit model that explains characteristics of SPOC at the single-sarcomere level. We further reduce the connected model to phase equations, revealing that the combination of local and global couplings is crucial to the emergence of these patterns.
\end{abstract}

Striated muscle is a biological force generator comprised of a highly periodic pattern of microstructures [Fig. 1(a)]. A basic structural unit is a half-sarcomere (for simplicity, here we call it a sarcomere), which is a barrel-like structure of 1-2 $\mu \mathrm{m}$ in size. The sarcomeres are connected in series to form a linear fiber named a myofibril. The contraction of each sarcomere occurs by the relative sliding of a set of two filaments, named the thick (myosin) and the thin (actin) filaments, which extend from viscoelastic proteinaceous structures, called the $M$ line and the $Z$ line, respectively [Fig. 1(b)]. Catalytic myosin "head" domains extending from the thick filament bind to the adjacent thin filament to form "cross bridges" [1,2] and generate the sliding force between the two filaments using the energy of ATP hydrolysis.

The force-generating activity of each sarcomere is, in general, controlled by the concentration of free $\mathrm{Ca}^{2+}$ : the sarcomeres develop force at high and relax at low $\mathrm{Ca}^{2+}$ concentrations. On the other hand, under fixed intermediate activation conditions, such as at $\mathrm{pCa} \sim 6.0$, a remarkable phenomenon, a spontaneous oscillatory contraction termed SPOC [3] occurs in both skeletal [4] and cardiac muscles [5,6]. During SPOC, each sarcomere repeats cycles of submicronsized length oscillations that are highly stable and comprised of slow shortening and rapid lengthening phases, associated with, respectively, the active force development and relaxation. The pattern of oscillation depends on several physical parameters, such as the number of sarcomeres in the myofibril and the level of activation [3-6]. The fact that SPOC occurs at the fixed chemical conditions suggests that the mechanical property of sarcomeres and local mechanical coupling between the sarcomeres play a crucial role [7].

The SPOC pattern is also affected by the external loading. When the load is maintained constant (isotonic condition), the oscillatory phase of each sarcomere is synchronized in phase [8]. Under auxotonic conditions, where the load is applied by the external spring [Fig. 1(a)], clear traveling (metachronal) waves along the myofibril are observed [3] [Fig. 1(d)]. When the total myofibril length is held constant (isometric condition), the traveling wave tends to be split into several parts [9] [Fig. 1(e)]. In some cases, the out-of-phase synchronization is observed [Fig. 1(f)]. These properties suggest that the global coupling via external load could also be essential. However, the consequences of the local and global couplings between sarcomeres remain largely uncharacterized.

There exist several theoretical models describing SPOC [10-13] that reproduce important features of sarcomere oscillation. The realistic model proposed by Smith and Stephenson [13] is based upon the elasticity of titin, a fibrous protein that connects the end of the thick filament and the $Z$ line. However, our experiments demonstrate that titin is not essential [3]. Other models are either abstract [10] or assume inertia of the external spring or friction from medium [11,12], the contribution of which is in fact very small compared to active and elastic forces. Moreover, more complex and realistic properties of SPOC as described above remain fully unexplained. We recently constructed a unit sarcomere model that can recapitulate the main characteristics of SPOC, including its sawtooth waveform and the phase diagram comprised of relaxation, contraction, and SPOC states $[3,14]$. In this Letter, we develop a mechanistic model of myofibrils by connecting the unit model and show that it can reproduce various oscillatory patterns of sarcomeres.

The unit model [14] is based on the following three principles: (i) Myosin heads stochastically and independently attach to or detach from the adjacent thin filament (two-state model), and the transition rates depend on the distance between the two filaments, i.e., the lattice spacing [15] [Figs. 1(b) and 1(c) and Eq. (1)]; (ii) the attached heads generate the active sliding force and, simultaneously, the friction [16], and the sum of these two forces is balanced with the externally applied load [Eq. (2)]; 

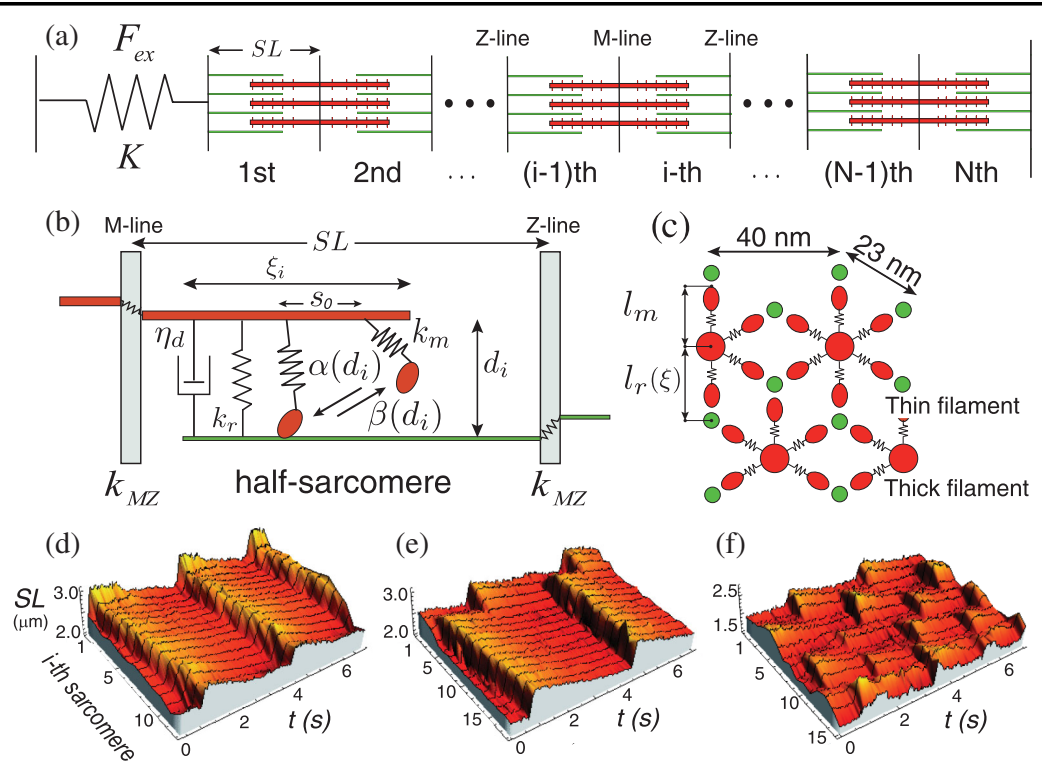

FIG. 1 (color). Schematic representation of a myofibril and a half-sarcomere. (a) Half-sarcomeres are connected alternatively in series across the $M$ and $Z$ lines. (b) The thick and thin filaments face each other and slide past one another during contraction. (c) Cross-sectional view of the overlapping zone of the thick and the thin filaments under the relaxing conditions. The lattice spacing, $l_{r}(\xi)$, is that at equilibrium in the absence of force-generating cross bridges. $l_{m}$ is an average natural length of the myosin head thermally fluctuating without attachment to the thin filament. (d), (e), (f) Representative patterns of SPOC waves-the traveling wave (d), the disrupted traveling wave (e), and the out-of-phase synchronization (f). The corresponding movies and experimental conditions are shown in the Supplemental Material, Movie 1 and Text 2, respectively [17].

(iii) the force balance in the short axis perpendicular to the myofibril's long axis is also taken into account [Eq. (4)]. [For more details on (ii) and (iii), see Supplemental Material, Text 1 [17]]. Since the parameters of the unit model describe the fundamental properties of the sarcomere structure, we can easily extend it to the myofibril model:

$$
\frac{d P_{i}}{d t}=\alpha\left(d_{i}\right)\left(1-P_{i}\right)-\beta\left(d_{i}\right) P_{i}
$$

Equation (1) is the time evolution equation for the fraction of myosin heads attached to the thin filament, $P_{i}$, which includes both the active force-generating state and the attached state that only contributes to passive force generation. Here, the subscript $i$ denotes quantities of the $i$ th sarcomere. Let the total number of sarcomeres be $N$. We assume that the attachment and detachment rates, $\alpha$ and $\beta$, depend on the lattice spacing $d_{i}$ of each sarcomere and take the forms $\alpha\left(d_{i}\right)=-\alpha_{1}\left(d_{i}-d_{0}\right) \Theta\left(d_{0}-d_{i}\right)$ and $\beta\left(d_{i}\right)=\beta_{0}$, where $d_{0}$ and $\beta_{0}$ are positive constants and $\Theta(x)$ is the Heaviside function defined as $\Theta(x)=1$ for $x>0$ and 0 otherwise. The slope of $\alpha(d), \alpha_{1}$, represents the level of activity of a myofibril, which in a physiological situation can be controlled by $\mathrm{Ca}^{2+}$ concentration: $\alpha_{1}=0$ corresponds to full relaxation, whereas sufficiently large $\alpha_{1}$ corresponds to full activation.

The force balance along the long axis of the myofibril is given by

$$
a \frac{\xi_{i}}{s_{0}} P_{i}-\eta_{m} \frac{\xi_{i}}{s_{0}} P_{i} \frac{d \xi_{i}}{d t}-F_{\mathrm{ex}}=0
$$

where $\xi_{i}$ is the length of overlap between the thick and thin filaments, which can be described as $S L_{i}=S L^{(0)}-\xi_{i}$ ( $S L_{i}$ is the length of the $i$ th sarcomere and $S L^{(0)}$ is the length at no overlap). Here, $\xi_{i} P_{i} / s_{0}$ is the total number of cross bridges, and $s_{0}$ is the interval between the adjacent myosin heads along each thick filament. The first and the second terms in Eq. (2) represent, respectively, the averaged active sliding force and the passive friction force generated by cross bridges [16], where $a$ and $\eta_{m}$ are positive constants (see the Supplemental Material, Text 1 for details [17]). All sarcomeres bear the same external force $F_{\text {ex }}$ because they are connected in series and both their inertia and the friction between sarcomeres and the surrounding medium (water) are negligible compared to the forces considered here. Whereas the form of $F_{\text {ex }}$ depends on the type of the external load (isotonic, auxotonic, or isometric), it is expressed as

$$
F_{\mathrm{ex}}=F_{0}+K \sum_{i=1}^{N}\left(\xi_{i}-\bar{\xi}\right)
$$

Here, $F_{0}$ is a given constant force, and $\bar{\xi}$ is a stationary solution of Eqs. (1), (2), and (4) for $\xi_{i}$ at $F_{\text {ex }}=F_{0} . K$ is a spring constant of the external spring, and the position of one end of the spring $X_{0}$ is given as $X_{0}=N\left(S L^{(0)}-\bar{\xi}\right)+$ $F_{0} / K$. The cases of $K \rightarrow 0$, a finite $K$, and $K \rightarrow \infty$ in Eq. (3) 
correspond to the isotonic, auxotonic, and isometric conditions, respectively.

The force balance along the short axis of a myofibril is given by

$$
\begin{gathered}
k_{r}\left(l_{r}\left(\xi_{i}\right)-d_{i}\right)+k_{m} P_{i} \frac{\xi_{i}}{s_{0}}\left(l_{m}-d_{i}\right) \\
-\eta_{d} \frac{d d_{i}}{d t}+V\left(d_{i-1}, d_{i}, d_{i+1}\right)=0 .
\end{gathered}
$$

The first term is the force generated by the filament lattice. We assume that the lattice has a linear stiffness $k_{r}$ and generates elastic force as it separates from the equilibrium spacing $l_{r}$ attained under the relaxing conditions [Fig. 1(c)]. It has been experimentally established that the interfilament distance at the equilibrium attained under the relaxing conditions decreases on increasing $S L$ [18]; therefore, we simply put $l_{r}\left(\xi_{i}\right)=l_{r 0}+l_{r 1} \xi_{i}$ with positive constants $l_{r 0}$ and $l_{r 1}$. The second term is the average force exerted by cross bridges, approximated by the elastic force produced by a linear spring with spring constant $k_{m}$ and natural length $l_{m}$ (for more details, see the Supplemental Material, Text 1 [17]). The third term $\eta_{d} d d_{i} / d t$ is the friction force due to the viscoelasticity of the filament lattice; $\eta_{d}$ is assumed to be small.

The function $V$ defines the strength of the interaction between the adjacent sarcomeres through the lattice spacing. By regarding the sarcomeres as being coupled mutually through the elastic springs with stiffness $k_{M Z}$ and assuming that the mechanical properties of $M$ and $Z$ lines are the same, we take the following form of $V$ :

$V\left(d_{i-1}, d_{i}, d_{i+1}\right)=k_{M Z}\left(\left(d_{i-1}-d_{i}\right)+\left(d_{i+1}-d_{i}\right)\right)$.

Summarizing all the facts noted above, Eqs. (1), (2), and (4) are rewritten in a concise form:

$$
\begin{aligned}
& \frac{d}{d t}\left(\begin{array}{c}
P_{i} \\
\xi_{i} \\
d_{i}
\end{array}\right)=\left(\begin{array}{c}
\alpha\left(d_{i}\right)\left(1-P_{i}\right)-\beta\left(d_{i}\right) P_{i} \\
\left(a \xi_{i} P_{i}-F_{0} s_{0}\right) / \eta_{m} \xi_{i} P_{i} \\
\left(k_{r}\left(l_{r}\left(\xi_{i}\right)-d_{i}\right)+k_{m} P_{i} \xi_{i}\left(l_{m}-d_{i}\right) / s_{0}\right) / \eta_{d}
\end{array}\right) \\
&+k_{M Z}\left(\begin{array}{c}
0 \\
0 \\
\left(d_{i+1}+d_{i-1}-2 d_{i}\right) / \eta_{d}
\end{array}\right) \\
& 0 \\
&+K\left(\begin{array}{c}
\sum_{j=1}^{N}\left(\bar{\xi}-\xi_{j}\right) s_{0} / \eta_{m} \xi_{i} P_{i} \\
0
\end{array}\right) .
\end{aligned}
$$

This equation includes both local and global couplings. Indeed, the second and third terms on the right-hand side become, respectively, the second derivative and the integration terms in some continuous limits. If we put $N=1, k_{M Z}=0, K=0$, and $\eta_{d} \rightarrow+0$, the connected model comes to the unit model.

The unit model shows supercritical Hopf bifurcation with the control parameters $\alpha_{1}$ (activation level) and $F_{0}$ (constant external force) [14]. If we set $\alpha_{1}$ and $F_{0}$ to any values within the oscillation region, aside from the vicinity of the bifurcation points, the system undergoes auto-oscillations showing sawtooth waveforms. In the subsequent analysis we use the values $\left(\alpha_{1}, F_{0}\right)=(19,20)$, but any set of values within the parameter region of the auto oscillation yields essentially the same results (for the units of time and length, see the Supplemental Material, Table 1 [17]).

In the connected model [Eq. (6)], the additional control parameters are $N$ and $K$. We take the Neumann boundary conditions expressed as $d_{0}=d_{1}$ and $d_{N+1}=d_{N}$. The initial condition is described in the legend of Fig. 3. The simulation time is much longer than the oscillation period to identify the final state of the system. If the final state is stationary, the phase of the $i$ th sarcomere $\phi_{i}$ is written as $\phi_{i}=\omega t+\hat{\phi}_{i}$, with constants $\omega$ and $\hat{\phi}_{i}$. We term $\hat{\phi}_{i}$ "the relative phase difference", and its profile identifies the type of the oscillation pattern. Figure 2 shows different oscillation patterns (the corresponding spatial profiles of $\hat{\phi}_{i}$ are shown in the Supplemental Material, Fig. 1 [17]).

The phase diagram for the oscillation patterns against $N$ and $K$ (Fig. 3) reveals several significant features. At small $K$ (the isotonic condition) the oscillatory units are in-phase synchronized, which is consistent with the experimental results [8]. At $K$ greater than the characteristic value $K^{*}$, this in-phase synchronization becomes unstable, and traveling waves emerge [Fig. 2(b)]. Since the locally coupled identical and linearly connected oscillators do not show traveling waves under Neumann boundary conditions
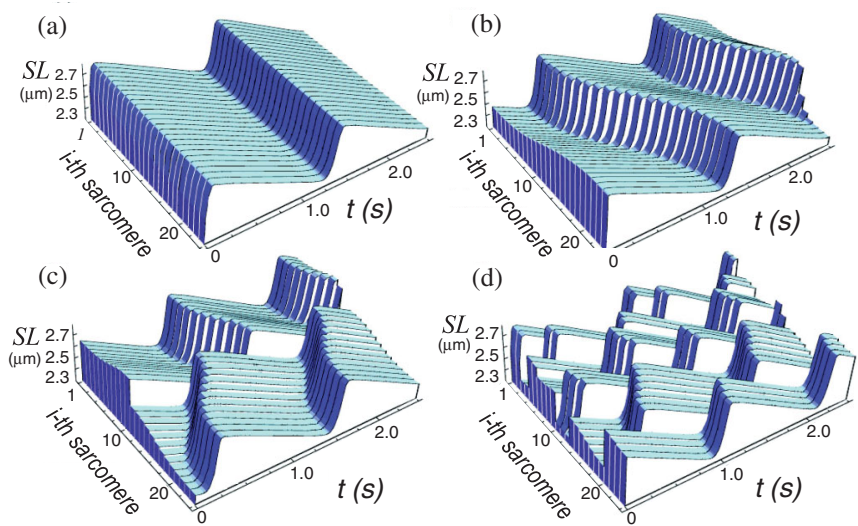

FIG. 2 (color). Examples showing typical spatiotemporal patterns of $S L$ oscillations. (a), (b), (c), and (d) show, respectively, in-phase synchronization, traveling waves, disrupted traveling waves, and out-of-phase synchronization. The values of $N$ and $K$ used in each simulation are $N=24$ and $K=0.01,0.18,0.7$, 3.75 , respectively, and the values of the other parameters are indicated in the legend of Fig. 3. It is to be noted that the SPOC period largely depends on $\eta_{m}$ as shown in Eq. (2), that is, the larger the value of $\eta_{m}$, the longer the SPOC period (see Fig. 8A in [14] for model simulation, and Figs. 3 and 4 in [3] for experimental results). The movies are shown in the Supplemental Material, Movie 2 [17]. 
[19], the appearance of traveling waves may be due to the contribution of global coupling. As $K$ increases, a different oscillatory pattern is observed, which we call "the disrupted traveling waves" [Fig. 2(c)]. Its characteristic is the existence of finite phase gaps between the traveling wave domains [Fig. 2(c) and see Supplemental Material, Fig. 1(c) [17]]. To the best of our knowledge, this oscillatory pattern has never been reported before in the system of coupled identical oscillators. As $K$ increases further, we enter the region of out-of-phase oscillation [Fig. 2(d)] and then contraction region (without oscillation) at any $N$ (Fig. 3). The connected model predicts the existence of nonstationary

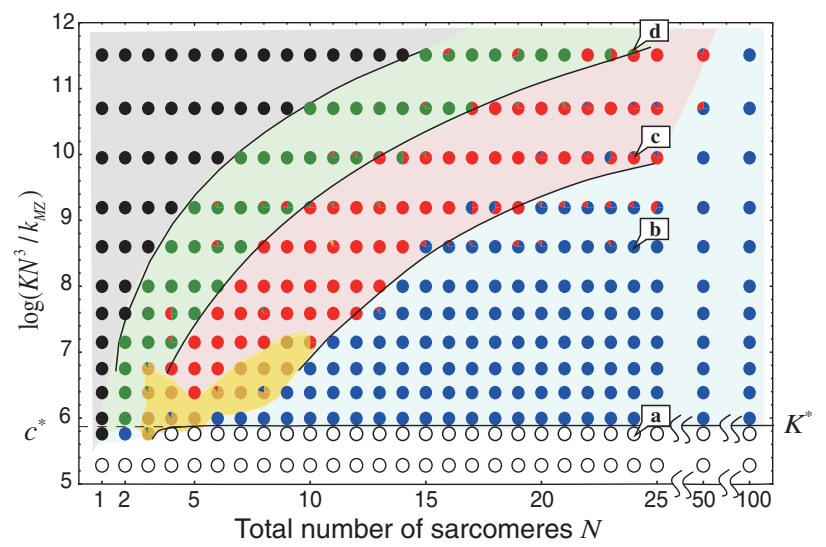

FIG. 3 (color). Phase diagram of the $S L$ oscillation patterns in the steady state under auxotonic conditions obtained from the connected model. Abscissa, the total number of sarcomeres, $N$. Ordinate, the ratio of the spring constant of the external spring, $K$, to the stiffness of the $Z$ (or $M$ ) line, $k_{M Z}, K N^{3} / k_{M Z}$. The color region indicates the steady state of $S L$ : in-phase synchronization (white), traveling wave (blue), disrupted traveling wave (red), outof-phase synchronization (green), nonstationary (yellow), and contraction without oscillation (black). Typical oscillation patterns corresponding to the markers (a)-(d) are shown in Fig. 2. In general, the final state depends on the initial conditions (e.g., $S L_{i}$ and its distribution), the dependence of which is indicated by the two-color circles where the proportion of each color indicates the probability of the appearance of the two states. The initial conditions used here are given by adding random numbers $\delta \in[-0.0001,0.0001]$ to a stationary solution $(\bar{P}, \bar{\xi}, \bar{d})=(0.18,1.08,25.1)$. When the initial randomness is increased to, for example, $\delta \in[-0.01,0.01]$, the contraction region (black) at larger $N$ values becomes the out-ofphase oscillations (green), while the other states are essentially unaltered. $c^{*}$ is $\log \left(-\pi^{2} B^{\prime}(0) / A^{\prime}(0)\right)$. The parameters are $\alpha_{1}=$ $19, F_{0}=20, \beta_{0}=20, k_{M Z}=0.5, \eta_{d}=0.0005, k_{m}=1, s_{0}=0.01$, $l_{m}=23, k_{r}=60, \eta_{m}=1.5, d_{0}=25.3, S L^{(0)}=3.6, l_{r 0}=23$, $l_{r 1}=2.53$, and $a=1$, which are nondimentionalized such that $k_{m}$ the force and length along the short axis $\left(1\right.$ (a.u.) $\mathrm{nm}^{-1}$ ) and $a$ the force along the long axis $(1 \mathrm{pN})$ are unity. In addition, the unit time is chosen as $1 \mathrm{~s}$, and the unit lengths for the short and long axes are chosen as $1 \mathrm{~nm}$ (for $d, l_{m}$, and $l_{r}$ ) and $1 \mu \mathrm{m}$ (for $s_{0}$ and $S L$ ), respectively. (For more details see the Supplemental Material, Table 1 [17].) The phase diagram is basically insensitive to the choice of the parameter values except that the disrupted traveling waves disappear when the parameters approach the Hopf bifurcation points. states at small $N$, which should be experimentally confirmed. With the exception of the yellow region in Fig. 3, all types of oscillatory patterns have been experimentally observed [3]. Several of those are shown in Figs. 1(d)-1(f), and the Supplemental Material [17].

To evaluate the results of numerical simulations of Eq. (6), we reduce the connected model to phase equations by means of the standard reduction method [20,21]. Let $\left(P^{(0)}(t), \xi^{(0)}(t), d^{(0)}(t)\right)$ be the $T$-periodic solution of Eq. (6) for $k_{M Z}=0$ and $K=0$, and $\phi_{i}\left(P_{i}, \xi_{i}, d_{i}\right)$ be the phase of the $i$ th sarcomere, satisfying $d \phi_{i} / d t=\omega_{0}=2 \pi / T$ in the absence of coupling $[20,21]$. The reduction theory states that if the interactions between the oscillatory units are sufficiently weak, the evolution equation of the coupled system is written only in terms of $\phi$ 's. Hence,

$$
\begin{aligned}
\frac{d \phi_{i}}{d t}= & \omega_{0}+k_{M Z}\left(B\left(\phi_{i}-\phi_{i+1}\right)+B\left(\phi_{i}-\phi_{i-1}\right)\right) \\
& +K \sum_{j=1}^{N} A\left(\phi_{i}-\phi_{j}\right) .
\end{aligned}
$$

$A$ and $B$ are coupling functions given by $A(\phi)=(1 / T) \times$ $\int_{0}^{T} d t Z_{2}(t)\left(\bar{\xi}-\xi^{(0)}\left(t-\phi / \omega_{0}\right)\right) s_{0} / \eta_{m} \xi^{(0)}(t) P^{(0)}(t)$ and $B(\phi)=(1 / T) \int_{0}^{T} d t Z_{3}(t)\left(d^{(0)}\left(t-\phi / \omega_{0}\right)-d^{(0)}(t)\right) / \eta_{d}$, where $Z_{2}(t)=\partial \phi /\left.\partial \xi\right|_{0}$ and $Z_{3}(t)=\partial \phi /\left.\partial d\right|_{0}$, with the symbol $\left.\bullet\right|_{0}$ denoting their values at $(P, \xi, d)=$ $\left(P^{(0)}(t), \xi^{(0)}(t), d^{(0)}(t)\right)$. The phase sensitivity $Z$ in the integrands of $A$ and $B$ are numerically calculated from the Malkin theory [22]. Equation (7) shows that only the relative coupling strength $K / k_{M Z}$ and the two coupling functions $A$ and $B$ determine the final state of Eq. (6). The explicit forms of $A$ and $B$ for the case $\left(\alpha_{1}, F_{0}\right)=(19,20)$ are given in Fig. 4. The signs of $B^{\prime}(0)$ (negative) and $A^{\prime}(0)$ (positive) indicate that with respect to small phase differences the local interaction is "attractive" and the global interaction is "repulsive." This result reflects that the lattice spacing in the adjacent sarcomeres tends to be equal and the external spring tends to keep the total myofibril length constant. To see how the value of $K^{*}$, above which the

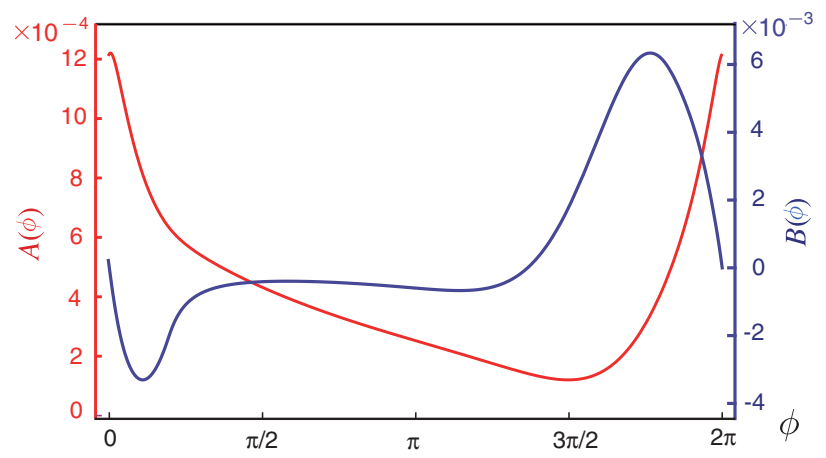

FIG. 4 (color). Phase-coupling functions obtained from Eq. (6). The parameters and the conditions are the same as in Fig. 3. $A(\phi)$ (red) and $B(\phi)$ (blue) are those for the global and local couplings, respectively. 
in-phase state is destabilized, is determined and how the traveling waves arise for $K>K^{*}$, we take a continuous limit in Eq. (7) assuming that $N$ is infinitely large. Introducing $x=i / N, \phi(x, t)=\phi_{i}(t), \hat{k}_{M Z}=k_{M Z} / N^{2}$ and $\hat{K}=K N$ into Eq. (7), we obtain

$$
\begin{aligned}
\frac{\partial \phi}{\partial t}= & \omega_{0}+\hat{k}_{M Z}\left(-B^{\prime}(0) \frac{\partial^{2} \phi}{\partial x^{2}}\right) \\
& +\hat{K} \int_{0}^{1} A\left(\phi(x, t)-\phi\left(x^{\prime}, t\right)\right) d x^{\prime},
\end{aligned}
$$

where we expand the local coupling in terms of $x$ derivatives of $\phi$ up to the leading term. $(\partial \phi / \partial x)^{2}$ has been dropped because $\left|B^{\prime \prime}(0)\right| \ll\left|B^{\prime}(0)\right|$. Linearizing Eq. (8) in $\phi$ gives $\partial \phi / \partial t=\left(\omega_{0}+\hat{K} A(0)\right)+\hat{k}_{M Z}\left(-B^{\prime}(0) \partial^{2} \phi / \partial x^{2}\right)+$ $\hat{K} A^{\prime}(0)\left(\phi(x, t)-\int_{0}^{1} \phi\left(x^{\prime}, t\right) d x^{\prime}\right)$, from which we obtain the critical value $K^{*}=-\pi^{2} k_{M Z} B^{\prime}(0) / N^{3} A^{\prime}(0)$ with the first destabilized mode $\cos \pi x$. The value of $K^{*}$ is in good agreement with that of the numerical simulations of Eq. (6) for large $N$ (Fig. 3). To find the stationary state for $K>K^{*}$, we write $\phi(x, t)$ as $\phi(x, t)=\omega t+\hat{\phi}(x)$ and substitute it into Eq. (8). This gives an integro differential equation for $\hat{\phi}: \partial^{2} \hat{\phi} / \partial x^{2}=\left[\hat{K} \int_{0}^{1} A\left(\hat{\phi}(x)-\hat{\phi}\left(x^{\prime}\right)\right) d x^{\prime}+\right.$ $\left.\left(\omega_{0}-\omega\right)\right] / \hat{k}_{M Z} B^{\prime}(0)$. To understand qualitatively the nature of the stationary solution of this equation, we tentatively put $A(\phi)=\sin \phi$ preserving the repulsiveness of $A$. Then, the above equation becomes equivalent to the equation of motion for a pendulum under gravitational force, if we regard $x$ as time. $\omega$ is given as $\omega=\omega_{0}$ and $\hat{\phi}(x)$ is given as the inverse of an elliptic function that is a monotonic function of $x$, implying a traveling wave. In the real case of $A$ containing higher harmonics, the solution is more complicated, but qualitatively remains the same: $\omega\left(>\omega_{0}\right)$ is uniquely determined, and $\hat{\phi}(x)$ is a monotonic function of $x$ [23].

In the present model, we assumed that the cross bridge is an independent force generator. Recently, Plaçais et al. reported that the actomyosin system composed of a small number of molecules can oscillate spontaneously under loading [24], suggesting that the actomyosin complexes function cooperatively [25]. Thus, it will be worth studying whether the cooperativity is involved in the fine mechanism of SPOC.

Here, we have demonstrated that the system where basic oscillatory units are connected in series has the localglobal coupling, producing various oscillation patterns. One point to be emphasized is that the common force acting on each unit works as a "repulsive global" coupling, and this type of interaction enhances the inhomogeneity of the system. In SPOC, this effect breaks the spatial uniformity of the system and, with the attractive local coupling, produces traveling or disrupted traveling waves. In the field of coupled oscillators, several studies [26] examined simultaneous local and attractive global couplings. Our observation that the mechanical coupling can as well produce repulsive global coupling may stimulate the investigation of this type of coupled systems.
We thank T. Kawakatsu, T. Shibata, S. Shima, N. Uchida, and Y. Hayakawa for their valuable comments and helpful support. We also thank Sergey V. Mikhailenko for his critical reading of manuscript. This work was supported by a RIKEN grant (to K. S.), and in part by Grants-in-Aid for Specially Promoted Research and Scientific Research (S) (to S. I.), from the MEXT Japan.

\section{*katsuhiko-sato@cdb.riken.jp}

†ishiwata@waseda.jp

[1] A.F. Huxley, Prog. Biophys. Biophys. Chem. 7, 255 (1957).

[2] H. E. Huxley, Science 164, 1356 (1969).

[3] S. Ishiwata, Y. Shimamoto, and N. Fukuda, Prog. Biophys. Molec. Biol. 105, 187 (2011).

[4] Y. Shimamoto, M. Suzuki, and S. Ishiwata, Biochem. Biophys. Res. Commun. 366, 233 (2008).

[5] A. Fabiato and F. Fabiato, J. Gen. Physiol. 72, 667 (1978).

[6] N. Fukuda, H. Fujita, T. Fujita, and S. Ishiwata, Pflügers Arch. 433, 1 (1996).

[7] Y. Shimamoto, M. Suzuki, S. V. Mikhailenko, K. Yasuda, and S. Ishiwata, Proc. Natl. Acad. Sci. U.S.A. 106, 11954 (2009).

[8] K. Yasuda, Y. Shindo, and S. Ishiwata, Biophys. J. 70, 1823 (1996).

[9] N. Okamura and S. Ishiwata, J. Muscle Res. Cell Motil. 9, 111 (1988).

[10] F. Julicher and J. Prost, Phys. Rev. Lett. 78, 4510 (1997).

[11] S. Gunther and K. Kruse, New J. Phys. 9, 417 (2007).

[12] A. Vilfan and T. Duke, Phys. Rev. Lett. 91, 114101 (2003).

[13] D. A. Smith and D. G. Stephenson, Biophys. J. 96, 3682 (2009).

[14] K. Sato, M. Ohtaki, Y. Shimamoto, and S. Ishiwata, Prog. Biophys. Molec. Biol. 105, 199 (2011).

[15] S. Ishiwata and F. Oosawa, J. Mechanochem. Cell Motil. 3, 9 (1974).

[16] K. Tawada and K. Sekimoto, J. Theor. Biol. 150, 193 (1991).

[17] See Supplemental Material at http://link.aps.org/ supplemental/10.1103/PhysRevLett.111.108104 for the forces generated by cross bridges (Text 1), experimental conditions (Text 2), the units of parameters (Table 1), and movies.

[18] B. M. Millman, Physiol. Rev. 78, 359 (1998).

[19] P. C. Bressloff and S. Coombes, Phys. Rev. Lett. 80, 4815 (1998).

[20] A. T. Winfree, J. Theor. Biol. 16, 15 (1967).

[21] Y. Kuramoto, Chemical Oscillations, Waves, and Turbulence (Springer, Berlin, 1984).

[22] B. Ermentrout, Neural Comput. 8, 979 (1996).

[23] We have also noted that the disrupted traveling wave reflects characteristic properties of $A$ and $B$ and their balance. The detailed study will be presented elsewhere.

[24] P.-Y. Plaçais, M. Balland, T. Guérin, J.-F. Joanny, and P. Martin, Phys. Rev. Lett. 103, 158102 (2009).

[25] S. Ishiwata, Y. Shimamoto, and M. Suzuki, HFSP J. 4, 100 (2010).

[26] D. Battogtokh and A. Mikhailov, Physica (Amsterdam) D 90, 84 (1996). 